\title{
Remembering Anna Piccotti
}

\section{Alfredo Musso ${ }^{1}$}

INFN - Sezione di Torino

1 via P. Giuria, 10125 Torino, Italy

E-mail: musso@to.infn.it

A few words to remember Anna Piccotti, a member of our RPC community who passed away in November 2011 as a result of car accident.

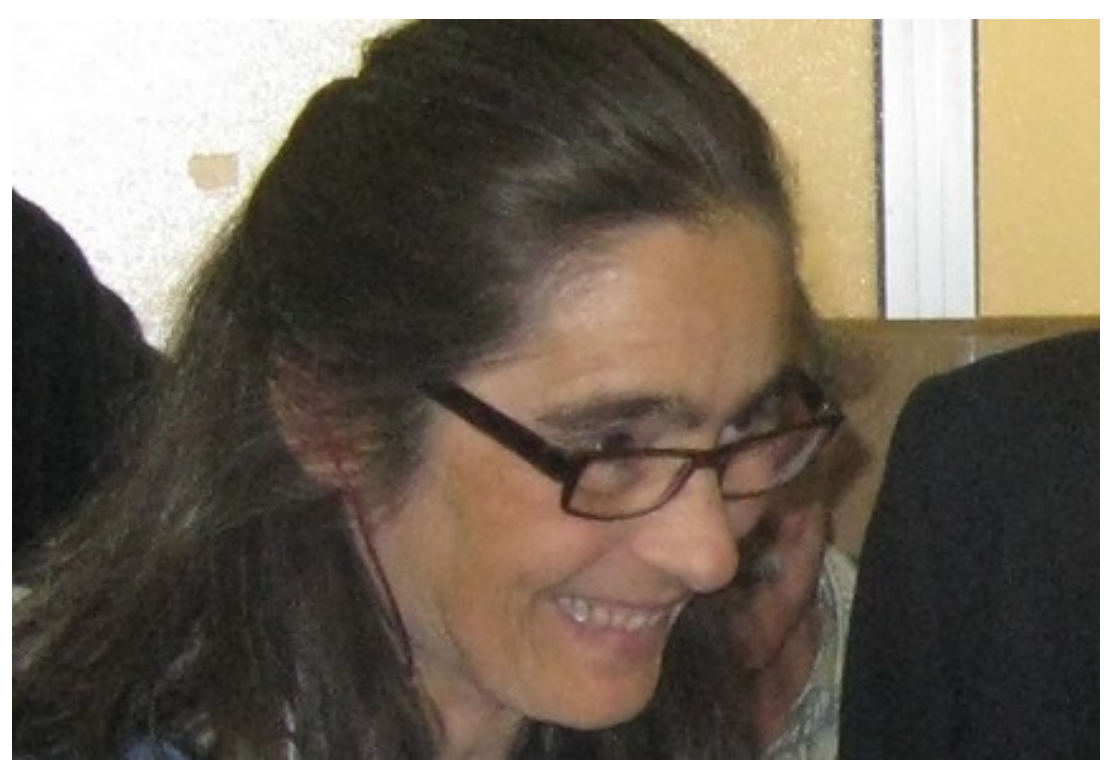

XI workshop on Resistive Plate Chambers and Related Detectors (RPC2012)

INFN-Laboratori Nazionali di Frascait, Italy

February 5-10, 2012

Speaker 


\section{In memoriam}

After her thesis in 1985 Anna Piccotti worked during almost 10 years on the PINOT Experiment at Saturne in Saclay aimed at the study of eta production on nuclear targets at $\sim 1 \mathrm{GeV}$ energies. At the beginning of 90's she participated to the PAN experiment at CERNLEAR to study spin structure in double charge exchange reactions. Successively she worked in NA50 experiment at CERN-SPS studying heavy ion interactions at ultra relativistic energies then entering in the ALICE Collaboration at CERN-LHC.

During the whole period she was employed at INFN-Sezione di Torino as "Ricercatore" at the beginning and then "Primo Ricercatore". She also tutored many students in the preparation of their Diploma and $\mathrm{PhD}$ thesis.

She was an excellent hardware-oriented physicist. In all these experiments she was responsible of various aspect of detector techniques: glass calorimeters, scintillators, limited streamer tubes, fiber sampling calorimeters and RPCs.

In the last decade she gave important contributions to the ALICE Zero-Degree Calorimeters and, in particular, she played a pivotal role in the construction, commissioning, maintenance and operation of the Resistive Plate Chambers of the Muon Trigger.

She also worked on R\&Ds for the application of RPCs in neutron detection and homeland security.

Anna participated to various RPC workshop since 1999 in Bari and was well known and appreciated in this community.

She was the detector-expert who followed all the phases of the Muon Trigger RPC project, from the R\&D to successful operation in ALICE and she was currently serving as a deputy project leader of the Muon Trigger system.

Anna died in a car accident on November 15, 2011.

All the colleagues who had the opportunity to work or to get in touch with Anna throughout the years have greatly appreciated her commitment and professional behavior, as well as her outstanding human qualities. She possessed a natural modesty as well as an easy and friendly personal contact with her colleagues.

This is a big loss for her family, for the Torino Group in which she worked during her entire 25 years career and for the RPC community. 